\title{
Regression and progression of microalbuminuria in adolescents with childhood onset diabetes mellitus
}

\author{
Mi Kyung Son, MD', \\ Ha Young Yoo, MD', \\ Byung Ok Kwak, MD ${ }^{1,2}$, \\ Hye Won Park, MD ${ }^{1,2}$, \\ Kyo Sun Kim, MD, PhD ${ }^{1,2}$, \\ Sochung Chung, MD, PhD ${ }^{1,2}$, \\ Hyun Wook Chae, MD ${ }^{3}$, \\ Ho-Seong Kim, MD, PhD ${ }^{3}$, \\ Duk Hee Kim, MD, PhD ${ }^{4}$
}

\footnotetext{
'Department of Pediatrics, Konkuk University Medical Center, Seoul, ${ }^{2}$ Department of Pediatrics, Konkuk University School of Medicine, Seoul, ${ }^{3}$ Department of Pediatrics, Severance Children's Hospital, Yonsei University College of Medicine, Seoul, ${ }^{4}$ Department of Pediatrics, Sowha Children's Hospital, Seoul, Korea
}

Purpose: Although microalbuminuria is considered as an early marker of nephropathy in diabetic adults, available information in diabetic adolescents is limited. The aim of this study was to investigate prevalence and frequency of regression of microalbuminuria in type 1 (T1DM) and type 2 diabetes mellitus (T2DM) patients with childhood onset.

Methods: One hundred and nine adolescents (median, 18.9 years; interquartile range (IQR), 16.5-21.0 years) with T1DM and 18 T2DM adolescents (median, 17.9 years; IQR, 16.8-18.4 years) with repeated measurements of microalbuminuria (first morning urine microalbumin/creatinine ratios) were included. The median duration of diabetes was $10.1(7.8-14.0)$ years and $5.0(3.5-5.6)$ years, respectively, and follow-up period ranged $0.5-7.0$ years. Growth parameters, estimated glomerular filtration rate, glycosylated hemoglobin ( $\mathrm{HbA} 1 \mathrm{c})$ and lipid profiles were obtained after reviewing medical record in each subject.

Results: The prevalence of microalbuminuria at baseline and evaluation were $21.1 \%$ and $17.4 \%$ in T1DM, and $44.4 \%$ and $38.9 \%$ in T2DM. Regression of microalbuminuria was observed in 13 T1DM patients (56.5\%) and 3 T2DM patients (37.5\%), and progression rate was $10.5 \%$ and $20 \%$ in T1DM and T2DM respectively. In regression T1DM group, HbA1C at baseline and follow-up was lower, and C-peptide at baseline was higher compared to persistent or progression groups. In T2DM, higher triglyceride was observed in persistent group.

Conclusion: Considerable regression of microalbuminuria more than progression in diabetes adolescents indicates elevated urinary microalbumin excretion in a single test does not imply irreversible diabetic nephropathy. Careful monitoring and adequate intervention should be emphasized in adolescents with microalbuminuria to prevent rapid progression toward diabetic nephropathy.

Keywords: Albuminuria, Type 1 diabetes mellitus, Type 2 diabetes mellitus, Child, Adolescent

\section{Introduction}

Microalbuminuria is known as an early marker of nephropathy and cardiovascular diseases in adults with diabetes ${ }^{1-4)}$ and hypertension ${ }^{5}$. In patients with childhood onset diabetes, the cumulative prevalence of microalbuminuria is about $25 \%-30 \%$ after 10 years of diabetes, and $40 \%-60 \%$ after 20 years of diabetes ${ }^{6,7)}$. International Society for Pediatric and Adolescent Diabetes, The American Diabetes Association and National Kidney Foundation recommend annual screening for microalbuminuria in children from 9 years of age with 5 years of insulin dependent diabetes mellitus (IDDM) duration, from 11 years of age with 2 years of IDDM duration, and adolescent with 2 years or more of IDDM duration ${ }^{8,99}$.

Regression of microalbuminuria was reported in type 1 diabetes adults that occurred in $58 \%$ of the patients with a 6-year cumulative incidence of microalbuminuria, and modifiable factors

Address for correspondence:

Sochung Chung, MD, PhD

Department of Pediatrics, Konkuk University Medical Center, Konkuk University School of Medicine, 120 Neungdong-ro, Gwangjin-gu, Seoul 143-729, Korea

Tel: +82-2-2030-7553

Fax: +82-2-2030-7748

E-mail: scchung@kuh.ac.kr 
were low glycosylated hemoglobin (HbAlc) levels, low blood pressure and low levels of lipid concentration ${ }^{10)}$. Regression of microalbuminuria over six years was also occurred in $31 \%$ of adults with type 1 diabetes in Denmark ${ }^{11)}$. In a pediatric study conducting type 1 diabetic children, cumulative incidence of microalbuminuria was $26 \%$ after 10 years of follow-up, of them $39 \%$ had regression to normoalbuminuria without recurrence ${ }^{6}$.

It was also reported that $72 \%$ of type 1 diabetic children had regression to normoalbuminuria using repeated random spot urine tests in a single center study ${ }^{12)}$. Therefore there is a focus on early detection and prevention of complications through routine screening and careful monitoring as well as adequate control of blood glucose and blood pressure in diabetic children ${ }^{12)}$.

In this study, we investigated prevalence and frequency of regression of microalbuminuria in adolescents with childhood onset type 1 (T1DM) and type 2 diabetes mellitus (T2DM).

\section{Materials and methods}

\section{Subjects}

A total of 127 patients (83 males and 44 females, 8-28 years) with childhood onset T1DM or T2DM, who visited department of pediatrics, Konkuk University Medical Center and Childhood Diabetes Clinic of Severance Children's Hospital were included from July 2007 to July 2014. One hundred and nine patients were T1DM and 18 patients were T2DM. Diabetic patients with macroalbuminuria or overt proteinuria, hematuria, menstrual bleeding, infections, fever, and other renal diseases were excluded. Confirmed cases of diabetic nephropathy treated with medication were also excluded in this study.

\section{Anthropometric and laboratory data measurements}

Height and body weight were measured with a stadiometer and a calibrated digital electric scale, and body mass index (BMI) was calculated in each subject. The $z$-scores of height, weight, and BMI were obtained by Lambda-Mu-Sigma (LMS) method $^{3,13)}$. Serum creatinine, HbAlc, C-peptide and lipid profiles were obtained. Estimated glomerular filtration rate (eGFR) was calculated using the Schwartz formula: eGFR (mL/ $\left.\mathrm{min} / 1.73 \mathrm{~m}^{2}\right)=\kappa \times$ height $(\mathrm{cm}) /$ serum creatinine $(\mathrm{mg} / \mathrm{dL})^{14)}$. The value of $\kappa$ was determined to be 0.55 for children aged between 2 and 13 years and adolescent girls, and 0.70 for adolescent boys.

First morning urine sample was obtained in all patients, with an average of 2.7 urine samples per a patient. Three or more urine samples were collected in $24.4 \%$ and 2 samples collected in $75.6 \%$ of the patients. Baseline was the time of initial measurement of microalbuminuria, not meant by the time of diagnosis as diabetes. Follow-up was the time of last measurement of microalbuminuria. Spot urine microalbumin/ creatinine ratio $(\mathrm{mg} / \mathrm{g})$ was measured by the Jaffe method, and microalbuminuria is defined as $30-300 \mathrm{mg} / \mathrm{g}$ of microalbumin/ creatinine ratio in spot urine ${ }^{15)}$. Persistent microalbuminuria was defined as the presence of microalbuminuria at baseline and

Table 1. Anthropometric and laboratory characteristics of patients with type 1 and type 2 diabetes

\begin{tabular}{|c|c|c|c|c|}
\hline \multirow{2}{*}{ Variable } & \multicolumn{2}{|c|}{$\operatorname{T1DM}(n=109)$} & \multicolumn{2}{|c|}{ T2DM ( $n=18)$} \\
\hline & Baseline & Follow-up & Baseline & Follow-up \\
\hline \multicolumn{5}{|l|}{ Anthropometric index } \\
\hline Age (yr) & $17.5^{\mathrm{a})}(15.1-20.5)$ & $18.9(16.5-21.0)$ & $15.4^{\text {a) }}(12.6-17.4)$ & $17.9(16.8-18.4)$ \\
\hline Sex, male/female & \multicolumn{2}{|c|}{$39 / 70$} & \multicolumn{2}{|c|}{$4 / 14$} \\
\hline Diabetes duration (yr) & $9.5^{\mathrm{a})}(6.5-13.5)$ & $10.1(7.8-14.0)$ & $0.9^{\mathrm{a})}(0.0-3.0)$ & $5.0(3.5-5.6)$ \\
\hline Height $(\mathrm{cm})$ & $162.0^{\mathrm{a})}(160.0-170.0)$ & $162.9(160.0-172.0)$ & $158.5^{\text {a) }}(153.5-160.5)$ & $160.2(153.5-163.2)$ \\
\hline Weight (kg) & $57.0^{\text {a) }}(53.0-64.0)$ & $58.5(54.0-65.0)$ & $61.0(54.0-73.7)$ & $63.4(54.0-75.5)$ \\
\hline $\mathrm{BMI}\left(\mathrm{kg} / \mathrm{m}^{2}\right)$ & $21.52^{\mathrm{a})}(19.53-23.15)$ & $21.91(20.03-23.41)$ & $24.74(21.09-30.88)$ & $24.31(21.09-29.02)$ \\
\hline Height z-score & $0.12^{\text {a) }}(-0.44$ to 1.19$)$ & $0.05(-0.55$ to 0.86$)$ & $0.50^{\mathrm{a})}(-0.15$ to 0.71$)$ & $-0.10(-1.45$ to 0.13$)$ \\
\hline Weight z-score & $0.31(-0.17$ to 1.00$)$ & $0.27(-0.17$ to 1.02$)$ & $1.02^{\mathrm{a})}(-0.01$ to 2.50$)$ & $0.35(-0.01$ to 2.17$)$ \\
\hline BMI z-score & $0.31(-0.43$ to 0.78$)$ & $0.30(-0.43$ to 0.83$)$ & $1.29(-0.02$ to 2.49$)$ & $0.99(-0.01$ to 2.27$)$ \\
\hline \multicolumn{5}{|l|}{ Biochemical profiles } \\
\hline Serum creatinine (mg/dL) & $0.80(0.70-0.90)$ & $0.75(0.69-0.85)$ & $0.80(0.60-0.90)$ & $0.65(0.60-0.76)$ \\
\hline eGFR $\left(\mathrm{mL} / \mathrm{min} / 1.73 \mathrm{~m}^{2}\right)$ & $125.7(110.0-134.6)$ & $127.9^{\mathrm{a})}(118.8-136.1)$ & $128.5(109.9-156.3)$ & $139.6(129.0-152.1)$ \\
\hline Urine microalbumin/creatinine (mg/g) & $9.4(6.3-26.6)$ & $13.3(8.6-24.8)$ & $25.3(11.5-56.0)$ & $17.7(6.2-42.0)$ \\
\hline Cholesterol (mg/dL) & $175.0(167.0-230.0)$ & $179.0(163.0-206.0)$ & $194.0(161.0-231.0)$ & $150.0(139.0-222.0)$ \\
\hline $\mathrm{TG}(\mathrm{mg} / \mathrm{dL})$ & $79.0(61.0-101.0)$ & $74.0(56.0-93.0)$ & $105.0(85.0-235.0)$ & $92.0(64.0-262.0)$ \\
\hline HDL-cholesterol (mg/dL) & $58.0(50.0-68.0)$ & $60.0^{a)}(52.0-68.0)$ & $49.5(43.0-52.0)$ & $53.0(45.0-59.0)$ \\
\hline $\operatorname{HbA1c}(\%)$ & $8.3(7.3-9.6)$ & $8.4(7.4-9.6)$ & $8.8(6.9-12.2)$ & $7.8(6.2-11.7)$ \\
\hline C-peptide (ng/mL) & $0.02(0.015-0.039)$ & $0.02(0.015-0.018)$ & $2.97^{\mathrm{a})}(2.471-3.733)$ & $1.96(1.432-2.393)$ \\
\hline
\end{tabular}

Values are presented as median (interquartile range) or number.

T1DM, type 1 diabetes mellitus; T2DM, type 2 diabetes mellitus; BMI, body mass index; eGFR, estimated glomerular filtration rate; TG, triglyceride; HDL-cholesterol, high density lipoprotein cholesterol; HbA1c, glycosylated hemoglobin; C-peptide, connecting peptide.

${ }^{a} P<0.005$ for baseline vs. follow-up assessments. 


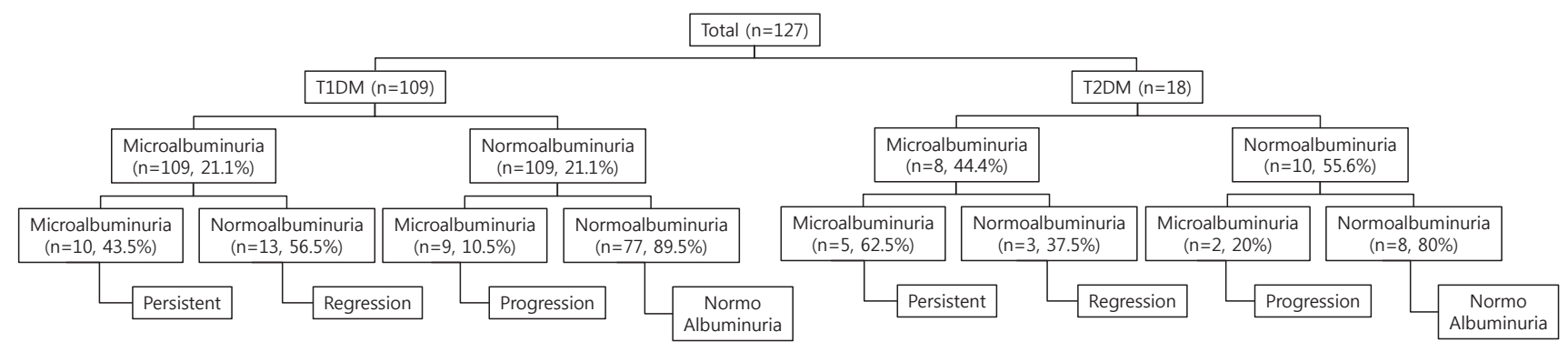

Fig. 1. Schematic representation of diabetic patients with persistent, regression, and progression groups to microalbuminuria. T1DM, type 1 diabetes mellitus; T2DM, type 2 diabetes mellitus.

Table 2. Characteristics of patients with type 1 diabetes according to microalbuminuria status $(n=109)$

\begin{tabular}{|c|c|c|c|c|c|c|c|c|}
\hline \multirow{2}{*}{ Characteristic } & \multicolumn{2}{|c|}{ Persistent $(n=10)$} & \multicolumn{2}{|c|}{ Regression $(n=13)$} & \multicolumn{2}{|c|}{ Progression $(n=9)$} & \multicolumn{2}{|c|}{ Normal $(n=77)$} \\
\hline & Baseline & Follow-up & Baseline & Follow-up & Baseline & Follow-up & Baseline & Follow-up \\
\hline \multicolumn{9}{|c|}{ Anthropometric index } \\
\hline Age $(y r)$ & $\begin{array}{c}17.8 \\
(14.5-22.5)\end{array}$ & $\begin{array}{c}19.2 \\
(17.0-23.0)\end{array}$ & $\begin{array}{c}17.0 \\
(15.0-21.0)\end{array}$ & $\begin{array}{c}20.0 \\
(17.5-21.5)\end{array}$ & $\begin{array}{c}19.0 \\
(19.0-24.5)\end{array}$ & $\begin{array}{c}20.0 \\
(19.5-25.0)\end{array}$ & $\begin{array}{c}17.5 \\
(15.0-20.5)\end{array}$ & $\begin{array}{c}18.0 \\
(15.5-21.0)\end{array}$ \\
\hline $\begin{array}{l}\text { Sex, } \\
\text { male/female }\end{array}$ & \multicolumn{2}{|c|}{$2 / 8$} & \multicolumn{2}{|c|}{$4 / 9$} & \multicolumn{2}{|c|}{$3 / 6$} & \multicolumn{2}{|c|}{$4 / 9$} \\
\hline $\begin{array}{l}\text { Diabetes } \\
\text { duration (yr) }\end{array}$ & $\begin{array}{c}12.0 \\
(7.5-16.0)\end{array}$ & $\begin{array}{c}12.8 \\
(8.0-16.5)\end{array}$ & $\begin{array}{c}10.0 \\
(5.0-11.1)\end{array}$ & $\begin{array}{c}10.5 \\
(7.9-13.0)\end{array}$ & $\begin{array}{c}11.5 \\
(5.5-15.0)\end{array}$ & $\begin{array}{c}12.0 \\
(7.5-16.0)\end{array}$ & $\begin{array}{c}9.5 \\
(6.5-13.5)\end{array}$ & $\begin{array}{c}10.0 \\
(7.0-14.0)\end{array}$ \\
\hline $\begin{array}{l}\text { Follow-up } \\
\text { duration (yr) }\end{array}$ & \multicolumn{2}{|c|}{$0.5(0.5-1.0) a)$} & \multicolumn{2}{|c|}{$1.0(0.5-4.0)$} & \multicolumn{2}{|c|}{$0.5(0.5-1.0) \mathrm{b})$} & \multicolumn{2}{|c|}{$1.0(0.5-0.5)$} \\
\hline Height z-score & $\begin{array}{c}0.10 \\
(-0.15-0.63)\end{array}$ & $\begin{array}{c}0.11 \\
(-0.15 \text { to } 0.53)\end{array}$ & $\begin{array}{c}-0.15 \\
(-0.41 \text { to } 0.28)\end{array}$ & $\begin{array}{c}-0.22 \\
(-0.55 \text { to } 0.26)\end{array}$ & $\begin{array}{c}0.01 \\
(-0.15 \text { to } 0.25)\end{array}$ & $\begin{array}{c}-0.01 \\
(-0.15 \text { to } 0.25)\end{array}$ & $\begin{array}{c}0.24 \\
(-0.55 \text { to } 1.02)\end{array}$ & $\begin{array}{c}0.15 \\
(-0.55 \text { to } 0.86)\end{array}$ \\
\hline Weight z-score & $\begin{array}{c}0.67 \\
(0.32-1.13)\end{array}$ & $\begin{array}{c}0.68 \\
(0.04-1.02)\end{array}$ & $\begin{array}{c}0.42 \\
(-0.10-0.71)\end{array}$ & $\begin{array}{c}0.22 \\
(-0.09 \text { to } 1.13)\end{array}$ & $\begin{array}{c}0.08 \\
(-0.02 \text { to } 0.79)\end{array}$ & $\begin{array}{c}0.08 \\
(-0.02 \text { to } 0.79)\end{array}$ & $\begin{array}{c}0.29 \\
(-0.17 \text { to } 0.90)\end{array}$ & $\begin{array}{c}0.29 \\
(-0.17 \text { to } 0.90)\end{array}$ \\
\hline BMI z-score & $\begin{array}{c}0.55 \\
(0.28-0.83)\end{array}$ & $\begin{array}{c}0.51 \\
(0.12-0.83)\end{array}$ & $\begin{array}{c}0.39 \\
(-0.12 \text { to } 0.76)\end{array}$ & $\begin{array}{c}0.34 \\
(0.30-0.68)\end{array}$ & $\begin{array}{c}-0.04 \\
(-0.47 \text { to } 0.77)\end{array}$ & $\begin{array}{c}-0.04 \\
(-0.11 \text { to } 0.77)\end{array}$ & $\begin{array}{c}0.28 \\
(-0.50 \text { to } 0.59)\end{array}$ & $\begin{array}{c}0.25 \\
(-0.50 \text { to } 0.59)\end{array}$ \\
\hline \multicolumn{9}{|c|}{ Biochemical profiles } \\
\hline $\mathrm{s} C r(\mathrm{mg} / \mathrm{dL})$ & $\begin{array}{c}0.70 \\
(0.60-0.90)\end{array}$ & $\begin{array}{c}0.69 \\
(0.65-0.74)\end{array}$ & $\begin{array}{c}0.80 \\
(0.70-1.00)\end{array}$ & $\begin{array}{c}0.73 \\
(0.69-0.99)\end{array}$ & $\begin{array}{c}0.78 \\
(0.60-0.80)\end{array}$ & $\begin{array}{c}0.75 \\
(0.74-0.81)\end{array}$ & $\begin{array}{c}0.80 \\
(0.70-0.90)\end{array}$ & $\begin{array}{c}0.76 \\
(0.69-0.86)\end{array}$ \\
\hline $\begin{array}{l}\mathrm{eGFR}(\mathrm{mL} / \\
\left.\mathrm{min} / 1.73 \mathrm{~m}^{2}\right)\end{array}$ & $\begin{array}{c}127.7 \\
(110.0-165.0)\end{array}$ & $\begin{array}{c}129.5 \\
(125.3-156.6)\end{array}$ & $\begin{array}{c}116.3 \\
(99.4-127.3)\end{array}$ & $\begin{array}{c}128.3 \\
(119.3-135.4)\end{array}$ & $\begin{array}{c}117.3 \\
(116.9-148.5)\end{array}$ & $\begin{array}{c}118.8 \\
(116.5-128.8)\end{array}$ & $\begin{array}{c}125.7 \\
(110.0-133.6)\end{array}$ & $\begin{array}{c}127.5 \\
(117.0-136.1)\end{array}$ \\
\hline $\begin{array}{l}\text { Urine } \\
\text { microalbumin/ } \\
\text { creatinine } \\
(\mathrm{mg} / \mathrm{g})\end{array}$ & $\begin{array}{c}63.3 \\
(48.9-171.8)\end{array}$ & $\begin{array}{c}58.0 \\
(48.0-92.4)\end{array}$ & $\begin{array}{c}56.4 \\
(34.1-74.0)\end{array}$ & $\begin{array}{c}9.6 \\
(8.6-15.8)\end{array}$ & $\begin{array}{c}15.4 \\
(11.4-22.0)\end{array}$ & $\begin{array}{c}42.1 \\
(30.8-50.2)\end{array}$ & $\begin{array}{c}7.4 \\
(5.0-10.1)\end{array}$ & $\begin{array}{c}11.3 \\
(7.8-16.5)\end{array}$ \\
\hline $\begin{array}{l}\text { Cholesterol } \\
(\mathrm{mg} / \mathrm{dL})\end{array}$ & NA & NA & $\begin{array}{c}175.0 \\
(160.0-214.0)\end{array}$ & $\begin{array}{c}168.0 \\
(163.0-206.0)\end{array}$ & NA & NA & $\begin{array}{c}181.0 \\
(175.0-187.0)\end{array}$ & $\begin{array}{c}177.5 \\
(160.0-195.0)\end{array}$ \\
\hline $\mathrm{TG}(\mathrm{mg} / \mathrm{dL})$ & $\begin{array}{c}70.0 \\
(64.0-135.0)\end{array}$ & $\begin{array}{c}75.0 \\
(68.0-119.0)\end{array}$ & $\begin{array}{c}79.5 \\
(67.0-109.0)\end{array}$ & $\begin{array}{c}77.5 \\
(73-94)\end{array}$ & $\begin{array}{c}92.0 \\
(81-94)\end{array}$ & $\begin{array}{c}71.0 \\
(61-89)\end{array}$ & $\begin{array}{c}76.0 \\
(56-99)\end{array}$ & $\begin{array}{c}67.0 \\
(53-91)\end{array}$ \\
\hline $\begin{array}{l}\text { HDL-cholesterol } \\
(\mathrm{mg} / \mathrm{dL})\end{array}$ & $\begin{array}{c}58.0 \\
(56.0-64.0)\end{array}$ & $\begin{array}{c}55.0 \\
(53.0-66.0)\end{array}$ & $\begin{array}{c}58.0 \\
(53.0-67.0)\end{array}$ & $\begin{array}{c}61.5 \\
(55.0-68.0)\end{array}$ & $\begin{array}{c}56.0 \\
(45.0-68.0)\end{array}$ & $\begin{array}{c}60.0 \\
(47.0-75.0)\end{array}$ & $\begin{array}{c}58.0 \\
(49.0-67.0)\end{array}$ & $\begin{array}{c}60.0 \\
(52.0-68.0)\end{array}$ \\
\hline $\mathrm{HbA1c}(\%)$ & $\begin{array}{c}9.8 \\
(8.7-13.8)^{c}\end{array}$ & $\begin{array}{c}9.7 \\
(8.9-10.1)^{d)}\end{array}$ & $\begin{array}{c}8.0 \\
(7.0-8.9)^{e)}\end{array}$ & $\begin{array}{c}8.0 \\
(7.1-9.4)^{f)}\end{array}$ & $\begin{array}{c}9.6 \\
(8.5-10.2)\end{array}$ & $\begin{array}{c}9.5 \\
(7.8-10.1)\end{array}$ & $\begin{array}{c}8.2 \\
(7.1-9.1)\end{array}$ & $\begin{array}{c}8.1 \\
(7.2-9.0)\end{array}$ \\
\hline $\begin{array}{l}\text { C-peptide } \\
\text { (ng/mL) }\end{array}$ & $\begin{array}{c}0.02 \\
(0.015-0.015)\end{array}$ & $\begin{array}{c}0.02 \\
(0.015-0.018)\end{array}$ & $\begin{array}{c}0.27 \\
(0.015-0.571)^{9)}\end{array}$ & $\begin{array}{c}0.02 \\
(0.020-0.050)\end{array}$ & $\begin{array}{c}0.03 \\
(0.015-0.100)^{h)}\end{array}$ & $\begin{array}{c}0.04 \\
(0.020-0.092)\end{array}$ & $\begin{array}{c}0.02 \\
(0.015-0.020)\end{array}$ & $\begin{array}{c}0.02 \\
(0.015-0.022)\end{array}$ \\
\hline
\end{tabular}

Values are presented as median (interquartile range or range) or number.

T1DM, type 1 diabetes mellitus; BMI, body mass index; sCr, serum creatinine; eGFR, estimated glomerular filtration rate; TG, triglyceride; $\mathrm{HDL}$-cholesterol, high density lipoprotein cholesterol; HbA1c, glycosylated hemoglobin; C-peptide, connecting peptide; NA, not available. ${ }^{\text {a) }} P<0.001$ for regression vs. persistent groups. ${ }^{\text {b) }} P=0.027$ for regression vs. progression groups. ${ }^{c} P=0.009$ for persistent, regression, progression and normoalbuminuria groups. ${ }^{d)} P=0.030$ for persistent, regression and progression groups. ${ }^{e} P=0.026$ for persistent vs. regression groups. ${ }^{\mathrm{f}} P=0.042$ for persistent vs. regression groups. ${ }^{g)} P=0.021$ for persistent vs. regression groups. ${ }^{\text {h) }} P=0.021$ for persistent vs. progression groups. 
follow up assessment. Regressed or transient microalbuminuria was defined as regression of microalbuminuria to normoalbuminuria during follow-up. Progression of microalbuminuria was defined as normoalbuminuria at baseline assessment followed by progression to microalbuminuria ${ }^{6)}$.

Medical record was reviewed retrospectively in each patient.

\section{Ethics and confidential data approvals}

This study was approved by the Institutional Review Board of Konkuk University Medical Center, Seoul, Korea (IRB No. KUH1090029).

\section{Statistical data analysis}

Data are expressed as median (interquartile range [IQR] or range), or number and proportion (\%). Standard descriptive statistics were used to summarize demographic and laboratory variables. Changes in variables between baseline and followup were tested using the Wilcoxon signed-rank test. MannWhitney $U$ test was used to compare between two groups, and Kruskal Wallis analysis of variance was used for multiple comparison. Statistical analyses were performed using IBM SPSS Statistics ver. 20.0 (IBM Co., Armonk, NY, USA). All reported $P$-values are two-tailed and $P$-values less than 0.05 were considered statistically significant.

Table 3. Characteristics of patients with type 2 diabetes according to microalbuminuria status $(\mathrm{n}=18)$

\begin{tabular}{|c|c|c|c|c|c|c|c|c|}
\hline \multirow{2}{*}{ Characteristic } & \multicolumn{2}{|c|}{ Persistent $(n=5)$} & \multicolumn{2}{|c|}{ Regression $(n=3)$} & \multicolumn{2}{|c|}{ Progression $(n=2)$} & \multicolumn{2}{|c|}{ Normoalbuminuria $(n=8)$} \\
\hline & Baseline & Follow-up & Baseline & Follow-up & Baseline & Follow-up & Baseline & Follow-up \\
\hline \multicolumn{9}{|c|}{ Anthropometric index } \\
\hline Age (yr) & $\begin{array}{c}14.9 \\
(13.3-16.8)\end{array}$ & $\begin{array}{c}17.9 \\
(17.8-18.1)\end{array}$ & $\begin{array}{c}14.1 \\
(11.9-26.0)\end{array}$ & $\begin{array}{c}18.2 \\
(13.8-27.0)\end{array}$ & $\begin{array}{c}18.9 \\
(15.8-22.0)\end{array}$ & $\begin{array}{c}19.7 \\
(16.8-22.5)\end{array}$ & $\begin{array}{c}14.4 \\
(12.3-17.4)\end{array}$ & $\begin{array}{c}17.0 \\
(15.8-18.4)\end{array}$ \\
\hline $\begin{array}{l}\text { Sex, } \\
\text { male/female }\end{array}$ & $0 / 3$ & $0 / 5$ & $0 / 2$ & $4 / 4$ & & & & \\
\hline $\begin{array}{l}\text { Diabetes } \\
\text { duration (yr) }\end{array}$ & $\begin{array}{c}2.6 \\
(0.2-3.0)\end{array}$ & $\begin{array}{c}5.6 \\
(5.3-6.6)\end{array}$ & $\begin{array}{c}0.0 \\
(0.0-13.0)\end{array}$ & $\begin{array}{c}4.1 \\
(1.9-14.0)\end{array}$ & $\begin{array}{c}2.3 \\
(0.5-4.0)\end{array}$ & $\begin{array}{c}3.0 \\
(1.0-5.0)\end{array}$ & $\begin{array}{c}0.7 \\
(0.0-3.0)\end{array}$ & $\begin{array}{c}4.6 \\
(3.2-5.1)\end{array}$ \\
\hline $\begin{array}{l}\text { Follow-up } \\
\text { duration (yr) }\end{array}$ & \multicolumn{2}{|c|}{$4.4(3.0-5.1)$} & \multicolumn{2}{|c|}{$1.9(1.0-4.1)$} & \multicolumn{2}{|c|}{$0.8(0.5-1.0)$} & \multicolumn{2}{|c|}{$2.6(0.5-5.0)$} \\
\hline Height z-score & $\begin{array}{c}0.00 \\
(-0.02 \text { to } 0.01)^{\mathrm{a})}\end{array}$ & $\begin{array}{c}-0.02 \\
(-1.45 \text { to }-0.01)\end{array}$ & $\begin{array}{c}-0.15 \\
(-0.55 \text { to } 0.05)\end{array}$ & $\begin{array}{c}-0.63 \\
(-0.79 \text { to }-0.15)\end{array}$ & $\begin{array}{c}-1.92 \\
(-2.23 \text { to }-1.60)\end{array}$ & $\begin{array}{c}-1.92 \\
(-2.23 \text { to }-1.61)\end{array}$ & $\begin{array}{c}0.65 \\
(0.46-0.98)\end{array}$ & $\begin{array}{c}0.12 \\
(-0.70 \text { to } 0.47)\end{array}$ \\
\hline Weight z-score & $\begin{array}{c}2.33 \\
(1.18-2.50)\end{array}$ & $\begin{array}{c}1.56 \\
(1.47-2.38)\end{array}$ & $\begin{array}{c}-0.15 \\
(-2.16 \text { to }-0.02)\end{array}$ & $\begin{array}{c}-0.02 \\
(-3.07-0.04)\end{array}$ & $\begin{array}{c}0.20 \\
(0.13-0.27)\end{array}$ & $\begin{array}{c}0.20 \\
(0.13-0.27)\end{array}$ & $\begin{array}{c}1.74 \\
(0.31-2.68)\end{array}$ & $\begin{array}{c}0.63 \\
(-0.01 \text { to } 2.29)\end{array}$ \\
\hline BMI z-score & $\begin{array}{c}2.10 \\
(1.28-2.85)\end{array}$ & $\begin{array}{c}2.27 \\
(1.57-2.35)\end{array}$ & $\begin{array}{c}-0.26 \\
(-2.39 \text { to }-0.02)\end{array}$ & $\begin{array}{c}-0.02 \\
(-2.97 \text { to } 0.49)\end{array}$ & $\begin{array}{c}1.16 \\
(1.00-1.31)\end{array}$ & $\begin{array}{c}1.13 \\
(0.94-1.31)\end{array}$ & $\begin{array}{c}1.83 \\
(-0.07 \text { to } 2.57)\end{array}$ & $\begin{array}{c}0.99 \\
(-0.16 \text { to } 2.27)\end{array}$ \\
\hline \multicolumn{9}{|c|}{ Biochemical profiles } \\
\hline $\mathrm{s} C r(\mathrm{mg} / \mathrm{dL})$ & $\begin{array}{c}0.80 \\
(0.58-0.90)\end{array}$ & $\begin{array}{c}0.59 \\
(0.49-0.64)\end{array}$ & $\begin{array}{c}0.80 \\
(0.52-1.10)\end{array}$ & $\begin{array}{c}0.62 \\
(0.55-0.76)\end{array}$ & $\begin{array}{c}0.63 \\
(0.60-0.65)\end{array}$ & $\begin{array}{c}0.71 \\
(0.66-0.76)\end{array}$ & $\begin{array}{c}0.85 \\
(0.65-0.90)\end{array}$ & $\begin{array}{c}0.75 \\
(0.61-0.81)\end{array}$ \\
\hline $\begin{array}{l}\mathrm{eGFR}(\mathrm{mL} / \\
\left.\mathrm{min} / 1.73 \mathrm{~m}^{2}\right)\end{array}$ & $\begin{array}{c}110.3 \\
(99.3-151.9)\end{array}$ & $\begin{array}{c}152.1 \\
(131.8-180.4)\end{array}$ & $\begin{array}{c}115.8 \\
(77.5-158.7)\end{array}$ & $\begin{array}{c}136.2 \\
(110.0-157.5)\end{array}$ & $\begin{array}{c}132.9 \\
(128.2-137.5)\end{array}$ & $\begin{array}{c}117.7 \\
(108.6-126.8)\end{array}$ & $\begin{array}{c}131.3 \\
(109.9-181.5)\end{array}$ & $\begin{array}{c}145.2 \\
(131.1-148.6)\end{array}$ \\
\hline $\begin{array}{l}\text { Urine } \\
\text { microalbumin/ } \\
\text { creatinine } \\
(\mathrm{mg} / \mathrm{g})\end{array}$ & $\begin{array}{c}63.48 \\
(40.18-117.46)\end{array}$ & $\begin{array}{c}68.59 \\
(42.04-72.26)\end{array}$ & $\begin{array}{c}56.00 \\
(34.29-241.43)\end{array}$ & $\begin{array}{c}15.29 \\
(13.23-20.02)\end{array}$ & $\begin{array}{c}6.21 \\
(3.55-8.88)\end{array}$ & $\begin{array}{c}38.76 \\
(30.20-47.32)\end{array}$ & $\begin{array}{c}13.16 \\
(9.29-21.32)\end{array}$ & $\begin{array}{c}5.96 \\
(3.83-9.13)\end{array}$ \\
\hline $\begin{array}{l}\text { Cholesterol } \\
(\mathrm{mg} / \mathrm{dL})\end{array}$ & $\begin{array}{c}218.0 \\
(194-253)\end{array}$ & $\begin{array}{c}209.0 \\
(147-338)\end{array}$ & $\begin{array}{c}196.0 \\
(161-231)\end{array}$ & $\begin{array}{c}174.0 \\
(160-188)\end{array}$ & NA & NA & $\begin{array}{c}162.0 \\
(136-165)\end{array}$ & $\begin{array}{c}139.0 \\
(136-141)\end{array}$ \\
\hline $\mathrm{TG}(\mathrm{mg} / \mathrm{dL})$ & $\begin{array}{c}295.0 \\
(179-581)^{b)}\end{array}$ & $\begin{array}{c}326.0 \\
(320-572)^{c)}\end{array}$ & $\begin{array}{c}87.0 \\
(85-251)\end{array}$ & $\begin{array}{c}95.0 \\
(60-149)\end{array}$ & $\begin{array}{c}181.0 \\
(127-235)\end{array}$ & $\begin{array}{c}215.0 \\
(168-262)\end{array}$ & $\begin{array}{c}68.5 \\
(55-106)\end{array}$ & $\begin{array}{c}64.0 \\
(60-82)\end{array}$ \\
\hline $\begin{array}{l}\text { HDL-cholesterol } \\
(\mathrm{mg} / \mathrm{dL})\end{array}$ & $\begin{array}{c}52.0 \\
(51-52)\end{array}$ & $\begin{array}{c}54.0 \\
(45-57)\end{array}$ & $\begin{array}{c}64.0 \\
(41-73)\end{array}$ & $\begin{array}{c}59.0 \\
(44-64)\end{array}$ & $\begin{array}{c}39.5 \\
(35-44)\end{array}$ & $\begin{array}{c}43.5 \\
(41-46)\end{array}$ & $\begin{array}{c}47.5 \\
(43-50)\end{array}$ & $\begin{array}{c}53.0 \\
(45-66)\end{array}$ \\
\hline $\mathrm{HbA1c}(\%)$ & $\begin{array}{c}11.2 \\
(7.0-12.2)\end{array}$ & $\begin{array}{c}11.1 \\
(8.2-12.3)\end{array}$ & $\begin{array}{c}13.3 \\
(7.6-15.0)\end{array}$ & $\begin{array}{c}6.3 \\
(6.1-12.1)\end{array}$ & $\begin{array}{c}6.7 \\
(6.1-7.3)\end{array}$ & $\begin{array}{c}7.8 \\
(6.4-9.2)\end{array}$ & $\begin{array}{c}8.3 \\
(5.6-11.1)\end{array}$ & $\begin{array}{c}6.6 \\
(5.4-11.7)\end{array}$ \\
\hline $\begin{array}{l}\text { C-peptide } \\
\text { (ng/mL) }\end{array}$ & $\begin{array}{c}3.73 \\
(2.710-3.750)\end{array}$ & $\begin{array}{c}2.25 \\
(1.960-3.100)\end{array}$ & $\begin{array}{c}2.08 \\
(1.010-2.230)\end{array}$ & $\begin{array}{c}2.43 \\
(1.420-3.440)\end{array}$ & $\begin{array}{c}3.41 \\
(2.470-4.340)\end{array}$ & $\begin{array}{c}1.83 \\
(1.270-2.390)\end{array}$ & $\begin{array}{c}3.00 \\
(2.970-3.200)\end{array}$ & $\begin{array}{c}1.63 \\
(1.180-2.130)\end{array}$ \\
\hline
\end{tabular}

Values are presented as median (interquartile range or range) or number.

T2DM, type 2 diabetes mellitus; BMI, body mass index; sCr, serum creatinine; eGFR, estimated glomerular filtration rate; TG, triglyceride; $\mathrm{HDL}$-cholesterol, high density lipoprotein cholesterol; $\mathrm{HbA1}$, glycosylated hemoglobin; C-peptide, connecting peptide; NA, not available. a) $P=0.014$ for persistent vs. regression, progression groups. ${ }^{b)} P=0.040$ for persistent, regression, progression and normoalbuminuria groups.

c) $P=0.012$ for persistent, regression, progression and normoalbuminuria groups. 


\section{Results}

One hundred and nine patients were T1DM (39 males and 70 females) and 18 patients were T2DM (4 males and 14 females). The median duration of diabetes was 10.1 years and 5.0 years, respectively (Table 1 ). Both T1DM and T2DM groups had shown the changes of anthropometric indexes between baseline and follow-up tests. In T2DM, BMI $z$-score had shown decreasing tendency, but there was no statistical significance. The median spot urine microalbumin/creatinine ratios at baseline and follow-up were $9.4 \mathrm{mg} / \mathrm{g}$ (IQR, 6.3-26.6 mg/g) and $13.3 \mathrm{mg} /$ $\mathrm{g}$ (IQR, 8.6-24.8 mg/g) in T1DM, and $25.3 \mathrm{mg} / \mathrm{g}$ (IQR, 11.5-56.0 $\mathrm{mg} / \mathrm{g})$ and $17.7 \mathrm{mg} / \mathrm{g}(\mathrm{IQR}, 6.2-42.0 \mathrm{mg} / \mathrm{g})$ in T2DM, but there were no statistical significance. The other profiles include $\mathrm{HbAlc}$ shown no statistical significance.

The prevalence of microalbuminuria at baseline and follow up were $21.1 \%$ and $17.4 \%$ in T1DM, and $44.4 \%$ and $38.9 \%$ in T2DM (Fig. 1). Patients were divided into 4 groups based on results of repeated microalbuminuria measurements; persistent, regression, progression of microalbuminuria and normoalbuminuria groups. In 109 T1DM patients, 23 patients had microalbuminuria at baseline, of them 13 (56.5\%) had regression of microalbuminuria, 10 (43.5\%) had persistent microalbuminuria, and 86 patients had no microalbuminuria at baseline, of them $9(10.5 \%)$ showed progression of microalbuminuria during follow-up period. In 18 T2DM patients, $3(37.5 \%)$ had regression of microalbuminuria, 5 (62.5\%) had persistent microalbuminuria, and $2(20.0 \%)$ had progression of microalbuminuria. Seventy-seven patients $(89.5 \%)$ in
T1DM and 8 patients $(80.0 \%)$ in T2DM had negative results of microalbuminuria during follow-up.

Clinical characteristics and laboratory parameters of T1DM and T2DM patients were compared among persistent, regression, progression, and normoalbuminuria groups (Tables $2,3)$. The duration of diabetes was not different among 4 groups in T1DM ( $P=0.353$ at baseline, $P=0.455$ at follow-up). In T2DM, no difference of duration of diabetes was also observed among 4 groups at baseline $(P=0.861)$, and at follow-up $(P=0.366)$. In T1DM, follow-up duration of microalbuminuria was longer in regression group compared to persistent $(P<0.001)$ or progression groups $(P=0.027)$. HbAlC at baseline $(P=0.009)$ and follow-up $(P=0.030)$ was lower in regression group compared to persistent or progression groups. C-peptide at baseline was higher in regression group compared to persistent group $(P=0.021)$. In T2DM, there was no significant difference between each group in anthropometric indexes except baseline height $z$-score $(P=0.014)$. And higher triglyceride (TG) was observed at both baseline $(P=0.040)$ and follow up $(P=0.012)$ in persistent group. Follow-up duration of microalbuminuria was not different significantly among T2DM groups $(P=0.297)$. Comparison of spot urine microalbumin/creatinine ratio between baseline and follow up was presented in Fig. 2 .

\section{Discussion}

The prevalence of microalbuminuria at baseline and followup were $21.1 \%$ and $17.4 \%$ in T1DM, and $44.4 \%$ and $38.9 \%$ in T2DM. The regression of microalbuminuria was observed in

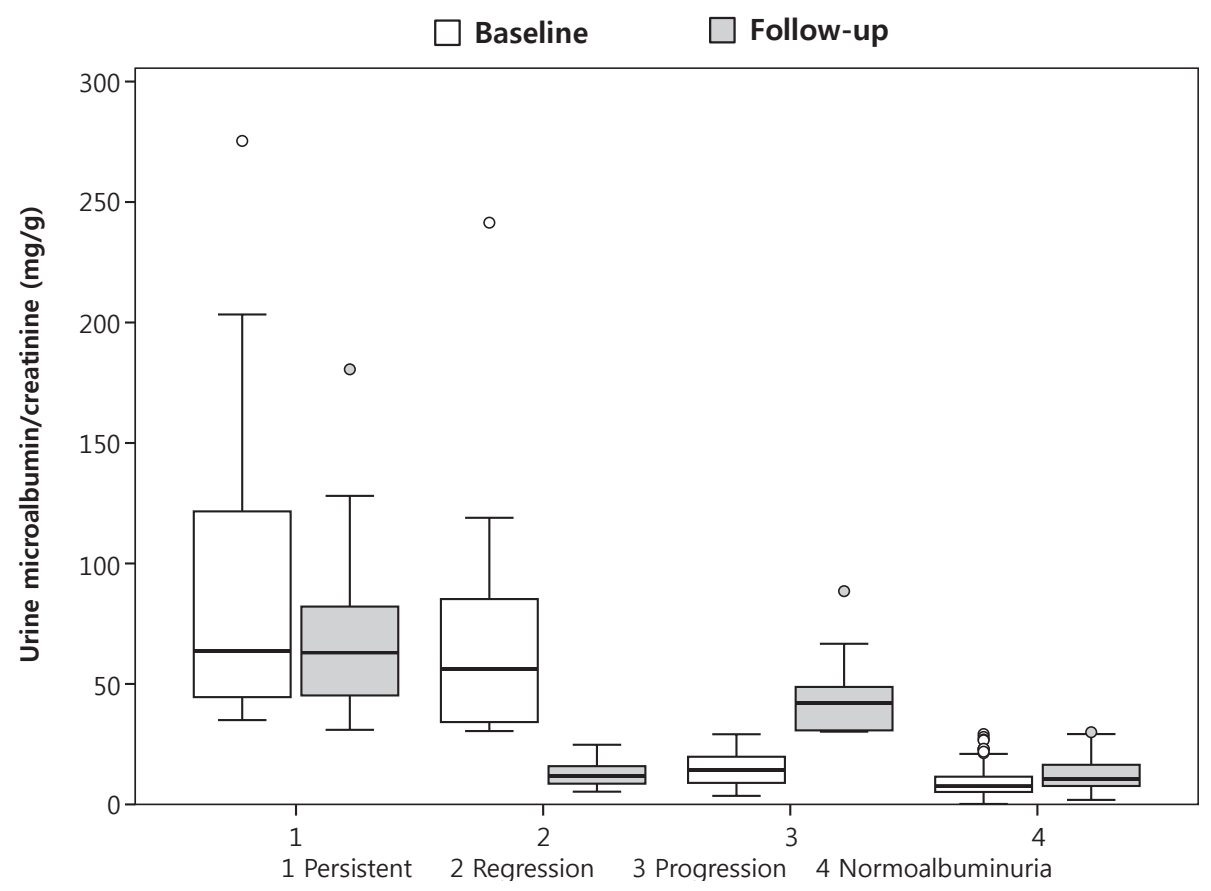

Fig. 2. Comparison of spot urine microalbumin/creatinine ratio between baseline and follow-up assessment. The median is depicted by the horizontal line, the interquartile range by the box limits, ranges by the whiskers, and outliers by the circles. 
$56.5 \%$ of T1DM patients and $37.5 \%$ of T2DM patients during follow-up. Lower baseline $\mathrm{HbAlc}$ (persistent vs. regression, $P=0.026$; progression vs. regression, $P=0.057$ ), higher C-peptide at baseline (persistent vs. regression, $P=0.021$ ) was observed in T1DM regression group compared to those in persistent or progression groups. In T2DM, patients in persistent group had higher TG compared to patients in other groups (baseline, $P=0.040$; follow-up, $P=0.012$ ).

While microalbuminuria was considered as a predictor of diabetic nephropathy in both adults and children ${ }^{2,3,16)}$, there have been studies which described regression of microalbuminuria in diabetic patients ${ }^{6,710,12,17-19)}$. Perkins et al. ${ }^{10)}$ found frequent regression of microalbuminuria, with $58 \%$ (95\% confidence interval, 52-64) incidence at the 6-year follow-up in T1DM adult patients. Low HbA1c, low blood pressure and low levels of lipid profiles were identified as modifiable factors. In the European multicenter study, 50.6\% of T1DM patients showed regression of microalbuminuria, whereas $13.9 \%$ showed progression over 7.3 years $^{20)}$. In the Oxford regional prospective study, the cumulative prevalence of microalbuminuria was $25.7 \%$ and $50.7 \%$ after 10 and 19 years of diabetes, of them $39 \%$ regressed to normoalbuminuria, while $13.9 \%$ progressed to macroalbuminuria ${ }^{6}$. Modifiable predictors of macroalbuminuria were high $\mathrm{HbAlc}$, and persistent and intermittent microalbuminuria. It was also reported that $72 \%$ of T1DM children had regression to normoalbuminuria using repeated spot urine tests in a single center study ${ }^{12)}$.

In patients with T2DM, microalbuminuria might be an early marker of T2DM, and it could precede a diagnosis of $\mathrm{T}_{2} \mathrm{DM}^{21,22)}$. Therefore, it was recommended for patients with T2DM to be screened for microalbuminuria at the beginning of diagnosis because the course of renal dysfunction is more heterogeneous, and the progression of nephropathy is less well characterized compared to that of $\mathrm{T}_{1 \mathrm{DM}}{ }^{17,23)}$. Moreover, the effect of medical treatment on diabetic nephropathy in T2DM has not been fully investigated. Until now, microalbuminuria in T2DM patients has been considered the first step toward overt proteinuria and nephropathy ${ }^{17)}$. However, recent studies reported that microalbuminuria in T2DM could be improved to normoalbuminuria ${ }^{17,24)}$. Araki et al. ${ }^{24)}$ found frequent remission and/or regression, with about 50\% incidence at the 6-year follow-up in T2DM adult patients. Short duration of microalbuminuria, the use of renin-angiotensin system blocking drugs, low $\mathrm{HbA1c}$ and low systolic blood pressure were identified as determinants. Gaede et al. ${ }^{25)}$ have reported that $30.5 \%$ of T2DM patients with microalbuminuria achieved remission to normoalbuminuria during 3-8 years, and antihypertensive therapy and low $\mathrm{HbAlc}$ were independent predictors for remission. In Japanese pediatric study, they presented that only $20 \%$ of children progressed to overt proteinuria, whereas $40 \%$ of children improved to normoalbuminuria among 130 T2DM patients with microalbuminuria during the followup years (mean \pm stdndard deviation, $3.4 \pm 1$.3 years; range, 2-6 years $)^{17)}$. Female sex, high HDL cholesterol and low
HbAlc were independently associated with remission and/ or regression of microalbuminuria. In this study, we identified frequent regression of microalbuminuria in $56.5 \%$ of T1DM and $37.5 \%$ of T2DM patients with a short follow-up period, which is consistent with previous studies ${ }^{6,10-12,17,24)}$. Duration of microalbuminuria, $\mathrm{HbAlc}$, blood pressure, lipid concentration and use of renin-angiotensin system blocking agents were well known risk factors of microalbuminuria ${ }^{26)}$. We found lower $\mathrm{HbA} 1 \mathrm{c}$ and higher C-peptide at baseline in regression group of T1DM, and higher TG in T2DM persistent group. It means that adequate control of glucose and lipid concentrations is important in diabetic children and adolescents, consistent with previous studies ${ }^{6,10,13,18,27-29)}$. Duration of microalbuminuria follow-up was longer in T1DM regression group compared to persistent or progression groups, while duration of diabetes did not differ among subgroups. It suggests the importance of frequent monitoring for microalbuminuria in diabetic children and adolescents. Early detection of microalbuminuria may lead to adequate intervention and, as a result, prevent progression toward diabetic nephropathy.

Our study has limitations. First, retrospective nature of study design is a limitation. Second, although important clinical and laboratory determinants of microalbuminuria were measured in the present study, other affecting factors such as blood pressure, sodium intake, and the use of angiotensin converting enzyme inhibitors or angiotensin receptor blocker were not included as a variable. Third, small sample size and short followup period is another limitation. During annual or biannual screening of microalbuminuria according to clinical practice guideline, frequent regression of microalbuminuria in both T1DM and T2DM patients was observed ${ }^{9)}$. Further studies including large number of childhood onset diabetes patients with long term follow-up period should be followed to identify contributing factors and mechanism of progression to overt proteinuria. Elevated microalbumin excretion is known to be an early marker of progression to diabetic nephropathy, however regression of microalbuminuria was observed in substantial number of patient. It indicates elevated urinary microalbumin excretion in a single test does not imply irreversible diabetic nephropathy. Close monitoring and intensive control should be emphasized in children and adolescents with microalbuminuria to prevent or delay the progression of diabetic nephropathy.

\section{Conflict of interest}

No potential conflict of interest relevant to this article was reported.

\section{References}

1. de Jong PE, Curhan GC. Screening, monitoring, and treatment of albuminuria: Public health perspectives. J Am Soc Nephrol 2006;17:2120-6.

2. Gerstein HC, Mann JF, Yi Q, Zinman B, Dinneen SF, Hoogwerf B, et al. Albuminuria and risk of cardiovascular 
events, death, and heart failure in diabetic and nondiabetic individuals. JAMA 2001;286:421-6.

3. Allen KV, Walker JD. Microalbuminuria and mortality in long-duration type 1 diabetes. Diabetes Care 2003;26:238991.

4. Bangstad HJ, Osterby R, Dahl-Jorgensen K, Berg KJ, Hartmann A, Nyberg G, et al. Early glomerulopathy is present in young, type 1 (insulin-dependent) diabetic patients with microalbuminuria. Diabetologia 1993;36:5239.

5. Ibsen H, Wachtell K, Olsen MH, Borch-Johnsen K, Lindholm LH, Mogensen CE, et al. Albuminuria and cardiovascular risk in hypertensive patients with left ventricular hypertrophy: the LIFE Study. Kidney Int Suppl 2004;(92):S56-8.

6. Amin R, Widmer B, Prevost AT, Schwarze P, Cooper J, Edge J, et al. Risk of microalbuminuria and progression to macroalbuminuria in a cohort with childhood onset type 1 diabetes: prospective observational study. BMJ 2008;336:697-701.

7. Kwon AR, Lee S, Chae HW, Kim DH, Kim HS. Frequencies and related factors for microvascular complications in patients with type 1 diabetes. Ann Pediatr Endocrinol Metab 2012;17:16-26.

8. Farmer AJ, Stevens R, Hirst J, Lung T, Oke J, Clarke P, et al. Optimal strategies for identifying kidney disease in diabetes: properties of screening tests, progression of renal dysfunction and impact of treatment - systematic review and modelling of progression and cost-effectiveness. Health Technol Assess 2014;18:1-128.

9. Donaghue KC, Wadwa RP, Dimeglio LA, Wong TY, Chiarelli F, Marcovecchio ML, et al. Microvascular and macrovascular complications in children and adolescents. Pediatr Diabetes 2014;15 Suppl 20:257-69.

10. Perkins BA, Ficociello LH, Silva KH, Finkelstein DM, Warram JH, Krolewski AS. Regression of microalbuminuria in type 1 diabetes. N Engl J Med 2003;348:2285-93.

11. Hovind P, Tarnow L, Rossing P, Jensen BR, Graae M, Torp I, et al. Predictors for the development of microalbuminuria and macroalbuminuria in patients with type 1 diabetes: inception cohort study. BMJ 2004;328:1105.

12. Montgomery KA, Ratcliffe SJ, Baluarte HJ, Murphy KM, Willi S, Lipman TH. Implementation of a clinical practice guideline for identification of microalbuminuria in the pediatric patient with type 1 diabetes. Nurs Clin North Am 2013;48:343-52.

13. Steinke JM, Sinaiko AR, Kramer MS, Suissa S, Chavers BM, Mauer M, et al. The early natural history of nephropathy in Type 1 Diabetes: III. Predictors of 5-year urinary albumin excretion rate patterns in initially normoalbuminuric patients. Diabetes 2005;54:2164-71.

14. Bogdanovic R. Diabetic nephropathy in children and adolescents. Pediatr Nephrol 2008;23:507-25.

15. Screening and management of microalbuminuria in patients with diabetes mellitus: recommendations to the
Scientific Advisory Board of the Bennett PH, Haffner S, Kasiske BL, Keane WF, Mogensen CE, Parving HH, et al. National Kidney Foundation from an ad hoc committee of the Council on Diabetes Mellitus of the National Kidney Foundation. Am J Kidney Dis 1995;25:107-12.

16. Dinneen SF, Gerstein HC. The association of microalbuminuria and mortality in non-insulin-dependent diabetes mellitus. A systematic overview of the literature. Arch Intern Med 1997;157:1413-8.

17. Ono T, Shikata K, Obika M, Miyatake N, Kodera R, Hirota $\mathrm{D}$, et al. Factors associated with remission and/or regression of microalbuminuria in type 2 diabetes mellitus. Acta Med Okayama 2014;68:235-41.

18. Salardi S, Balsamo C, Zucchini S, Maltoni G, Scipione M, Rollo A, et al. High rate of regression from micromacroalbuminuria to normoalbuminuria in children and adolescents with type 1 diabetes treated or not with enalapril: the influence of HDL cholesterol. Diabetes Care 2011;34:424-9.

19. Svensson M, Eriksson JW, Dahlquist G. Early glycemic control, age at onset, and development of microvascular complications in childhood-onset type 1 diabetes: a population-based study in northern Sweden. Diabetes Care 2004;27:955-62.

20. Giorgino F, Laviola L, Cavallo Perin P, Solnica B, Fuller J, Chaturvedi N. Factors associated with progression to macroalbuminuria in microalbuminuric Type 1 diabetic patients: the EURODIAB Prospective Complications Study. Diabetologia 2004;47:1020-8.

21. Alberti KG, Zimmet PZ. Definition, diagnosis and classification of diabetes mellitus and its complications. Part 1: diagnosis and classification of diabetes mellitus provisional report of a WHO consultation. Diabet Med 1998;15:539-53.

22. Maahs DM, Snively BM, Bell RA, Dolan L, Hirsch I, Imperatore $\mathrm{G}$, et al. Higher prevalence of elevated albumin excretion in youth with type 2 than type 1 diabetes: the SEARCH for Diabetes in Youth study. Diabetes Care 2007;30:2593-8.

23. Gambara V, Mecca G, Remuzzi G, Bertani T. Heterogeneous nature of renal lesions in type II diabetes. J Am Soc Nephrol 1993;3:1458-66.

24. Araki S, Haneda M, Sugimoto T, Isono M, Isshiki K, Kashiwagi A, et al. Factors associated with frequent remission of microalbuminuria in patients with type 2 diabetes. Diabetes 2005;54:2983-7.

25. Gaede P, Tarnow L, Vedel P, Parving HH, Pedersen O. Remission to normoalbuminuria during multifactorial treatment preserves kidney function in patients with type 2 diabetes and microalbuminuria. Nephrol Dial Transplant 2004;19:2784-8.

26. Roett MA, Liegl S, Jabbarpour Y. Diabetic nephropathy: the family physician's role. Am Fam Physician 2012;85:883-9.

27. Stone ML, Craig ME, Chan AK, Lee JW, Verge CF, Donaghue KC. Natural history and risk factors for microalbuminuria 
in adolescents with type 1 diabetes: a longitudinal study. Diabetes Care 2006;29:2072-7.

28. de Boer IH; DCCT/EDIC Research Group. Kidney disease and related findings in the diabetes control and complications trial/epidemiology of diabetes interventions and complications study. Diabetes Care 2014;37:24-30.

29. Chiarelli F, Trotta D, Verrotti A, Mohn A. Treatment of hypertension and microalbuminuria in children and adolescents with type 1 diabetes mellitus. Pediatr Diabetes 2002;3:113-24. 